\title{
Golden jubilee monograph
}

J. H. S. Blaxter

The Eggs and Planktonic Stages of British Marine Fishes. By Sir Frederick S. Russell. Pp. xv+524. (Academic; London, New York, San Francisco, 1976.) $£ 19.50$.

THERE is a tendency, as fisheries come under increasing pressure, for the success of the fishery to become dependent on the recruit fish rather than on a longer run of successful year-classes. The study of the pre-recruit phases of the life history, both for the purpose of prediction as well as for understanding what determines success or failure of a year-class, then assumes greater importance. This is one of the reasons why investigations of eggs, larvae and juveniles of commercial fish species have been greatly stepped up over the past twenty years. Both sea surveys of these young stages, as well as laboratory experiments involving rearing from the egg, are now part of the standard repertoire of most larger fisheries laboratories, and it is now generally accepted that the year-class strength is probably determined in the first year of life.

It is surprising that no standard work of identification of the young stages of north-eastern Atlantic species has been written since Ehrenbaum's Eier und Larven von Fischen which formed the first volume of his Nordisches Plankton, produced between the years 1905 and 1909. Many imperfections in identification existed at that time. To a limited extent they were made good in subsequent publications on restricted groups of species such as the gadoids by Johannes Schmidt in 1909, or in more comprehensive descriptions for other areas, as in the Fauna and Flora of the Gulf of Naples during the 1930s and in the ICES plankton identification sheets that appeared after World War II.

Sir Frederick Russell's book thus fulfills a clear need. It is a first class and completely fresh re-appraisal of the identification problem. It covers the planktonic stages of coastal species of northern European waters likely to be found within the 200-m depth contour but it excludes strictly oceanic species. Introductory chapters on terminology, development, principles of identification, feeding habits and vertical and horizontal distribution are followed by the main section where each teleost family is taken in turn. A description, with key where appropriate, is given for the egg, larva and post-larval stage. Relevant ecological or experimental work is also referred to-one aspect of the book which makes it quite different from previous publications.

The value of the monograph lies therefore in the collation of the taxonomic and other publications, and the assembling and up-dating of the many keys scattered in the literature. During this process the author has brought to bear his unique experience in this field, to improve, clarify and amplify the keys.

The reference to many 1975 and even some 1976 scientific papers in the bibliography is evidence of his success in making the monograph as comprehensive and up-to-date as possible within the range of species adopted. It is, however, a pity that the style and content of the book, limited as it is to northern European fishes, mean that some of the excellent and relevant ecological and experimental work done, for example, on the Californian sardine and anchovy, has perforce had to be excluded.

Sir Frederick Russell retired from the directorship of the Plymouth laboratory in 1969 and this monograph is a tour de force representing the results of his enviable energy during subsequent years. He published his first paper on fish eggs and larvae in 1926 so that the monograph is a fitting and admirable golden jubilee offering to the scientific community. We all look forward to the celebration of his diamond jubilee!

Dr Blaxter is a Senior Principal Scientific Officer at Dunstaffnage Marine Research Laboratory, Oban, Argyll, UK.

\section{Energetic ion beams and sputtering}

Ion Implantation, Sputtering and Their A pplications. By P. D. Townsend, J. C. Kelly and N. E. W. Hartley. Pp. ix + 333. (Academic: London and New York, June 1976.) £13; \$32.25.

IN a great many practical situations the behaviour of a component depends on the near-surface composition, physical state or topography of the material. Examples spring to mind in the optical, mechanical, electrical and corrosion fields, and the extraordinary developments in semiconductor devices and circuits involve a sophisticated control of material properties within a layer a few microns thick.

Diffusion, coating and etching have hitherto been used to control surface composition and its lateral variation, but these are no longer precise, versatile or reproducible enough for some requirements. The past decade has seen the development of an entirely different set of techniques based on the use of energetic ion beams by means of which chosen atomic species can be injected into a surface, or, by sputtering, material can be removed.

This book provides a very timely review of the progress achieved so far in a field in which industrial applications are being extended as each month goes by. The fundamentals of the subject are treated admirably, from the slowing-down of the incident ions to their effects on the target structure. The treatment of applications is more superficial, but the authors must be conscious of the speed with which a detailed account would become outdated. Readers, and there will be many, who are mainly interested in the ion implantation of semiconductors will be disappointed: the literature of this is now so extensive that the authors, perhaps wisely, have chosen to concentrate on the remainder.

A bonus, for which the reader is not well prepared by the title of the book, is a lengthy and most useful chapter dealing with the analytical applications of ion beams.

1 congratulate the authors on their clear and readable treatment, and the publisher for the attractive presentation of text and diagrams.

G. Dearnaley

Dr Dearnaley is a member of the research staff at the Atomic Energy Research Establishment, Harwell, UK. 